\title{
Thermal analysis of fibres and films in solvents and vapours ${ }^{1}$
}

\author{
Duncan M. Price* \\ Courtaulds plc.,101 Lockhurst Lane, Coventry CV6 5RS, U.K.
}

\begin{abstract}
The thermomechanical and dynamic mechanical properties of films and fibres in vapour laden atmospheres and simulated dye baths were investigated in order to understand their processing behaviour. Acetone and water vapour acted as plasticisers for cellulose acetate film. 2-phenoxyethanol was found to penetrate poly(ethylene terephthalate) film above its glass transition temperature and consequently depress its glass transition temperature. The use of 'carriers' to enhance the rate of dyeing of acrylic fibre was investigated. The increase in coloration was found to be proportional to the degree of plasticisation caused by the candidate compounds. () 1997 Elsevier Science B.V.
\end{abstract}

Keywords: Acrylic fibre; Cellulose acetate; Dynamic mechanical analysis; Dyeing; Poly(ethylene terephthalate); Thermomechanical analysis

\section{Introduction}

Many polymers come into contact with solvents or their vapours during processing; this may be intentional (e.g. during washing or dyeing operations) or unintentional (through insufficient drying or solvent recovery). Measurement of their thermal properties in such an environment gives an insight into the way performance can be modified by exposure to potential plasticisers. Thermomechanical and dynamic mechanical measurements (TMA and DMA) are the most appropriate methods to monitor the behaviour of polymers in liquids or saturated vapours because they avoid problems due to condensation or evaporation which plague calorimetric studies. Often, the investigator wishes to know what effect the environmental conditions can have on the stiffness and strength of the product. As will be illustrated, TMA and DMA are also more suited to making measurements under 'industrial' conditions.

One of the earliest studies in this area was the work of Meldrum \& Ward [1] who measured the compressibility of a pack of wet acrylic fibre as a function of temperature as part of a study of their dyeing behaviour and thermoplastic properties in relation to practical dyeing operations. Their data indicates a glass-rubber transition $\left(\mathrm{T}_{\mathrm{g}}\right)$ in the region $70-80^{\circ} \mathrm{C}$ as opposed to the normal dry $\mathrm{T}_{\mathrm{g}}$ of 90- $100^{\circ} \mathrm{C}$. Rosenbaum [2] employed linear thermal expansion, creep and dye sorption measurements to show that water reduced the $\mathrm{T}_{\mathrm{g}}$ of acrylic fibres by $30-35^{\circ} \mathrm{C}$. Murayama \& Armstrong [3] and Desai \& Wilkes [4] appear to have independently developed methods for measuring the dynamic mechanical properties of fibres and films in liquid environments using a modified Rheovibron Viscoelastometer. This was used to study Nylon 66 filaments in deionised water, tetrachloroethylene \& dry air and poly(ethylene terephthalate) (PET) film in the presence of various solvents. Ingamells \& Yanumet [5] used the same technique on PET to support the claim that aromatic and cyclic solvents solvate the aromatic part of the repeat unit and aliphatic solvents solvate the aliphatic part. Similar studies were carried out by Padhye \& Karandikar [6] on

\footnotetext{
*Corresponding author. Fax: +44 (0) 1203 638238; e-mail: duncan.m.price@ corp.courtaulds.co.uk.

${ }^{1}$ Presented at the First UK National Symposium on Thermal Analysis and Calorimetry, Leeds 17-18 April 1996
} 
polyacrylonitrile fibres to show plasticisation and anti-plasticisation by aromatic and non-aromatic solvents respectively.

This paper considers three areas of practical importance in the dying and finishing of fibres and films. Firstly, the handling of dry spun cellulose acetate filaments is thought to be affected by the amount of retained solvent (acetone) and relative humidity around the plant. Another area of interest is the deep dyeing of PET film which is currently done using an ethylene glycol dye bath [7]. 2-phenoxyethanol $\left(\mathrm{C}_{6} \mathrm{H}_{5} \mathrm{OCH}_{2} \mathrm{CH}_{2} \mathrm{OH}\right)$ has been considered as an alternative dye bath medium but, apart from the work by Ingamells and Yanumet [5], little was known on its effects on this material. Finally, the effect of 'carriers' - additives to the dye bath which are used to accelerate the rate of dyeing in acrylic and other hydrophobic fibres - was studied. One possible mechanism by which these materials enhance dye uptake is by plasticising the fibre thus increasing the segmental mobility of the polymer chains $[8,9]$.

The objectives of this work were therefore to develop reliable techniques for studying the thermal behaviour, most notably the glass transition temperature, of fibres and films under the effects of various solvents and vapours.

\section{Experimental}

Cellulose acetate film was cast from acetone solution onto a glass plate under conditions of controlled humidity $\left(0 \% \mathrm{RH}\right.$ and $100 \% \mathrm{RH}$ at $\left.20^{\circ} \mathrm{C}\right)$ and acetone vapour concentration (saturated at $20^{\circ} \mathrm{C}$ ). Poly (ethylene terephthalate) film (Melinex) was obtained from ICI. Undyed acrylic fibre (Courtaulds Courtelle $\mathrm{S}$ ) was cleaned in a non-anionic surfactant and washed to remove surface impurities. Bundles of fibre were dyed at $80^{\circ} \mathrm{C}$ for 30 minutes in a liquor containing dye (CI Basic Blue 3) and carrier solution buffered to $\mathrm{pH} 4.5$. After dyeing the samples were cooled and washed in warm water and acetone to remove excess dye and carrier before drying in an oven at $40^{\circ} \mathrm{C}$. Their dye uptake was measured by dissolving a sample of material in glacial acetic acid/dimethyl formamide $(20 \% \mathrm{v} / \mathrm{v})$. The absorbance of the solution was then measured at $649 \mathrm{~nm}$ (the $\ddot{e}_{\max }$ of the dyestuff in this medium) using a Pye-Unicam PU8600 UV/visible spectrophotometer and the concentration of the dye in the fibre determined with reference to the extinction coefficient of the dye and concentration of the solution. Fibre samples for $T_{g}$ determination were prepared in the same way without the addition of dye to the treatment bath. The fibre was not dried, but allowed to remain in the residual liquor until analysis.

Thermomechanical Analysis of cellulose acetate films was carried out on a Mettler TMA 40 using the quartz film \& fibre extension accessory supplied by the manufacturer. Measurements were made under dynamic loading conditions of $\pm 0.05 \mathrm{~N}$ at $1 / 12 \mathrm{~Hz}$ superimposed on a static force of $0.1 \mathrm{~N}$ at a heating rate of $2^{\circ} \mathrm{C} \mathrm{min}^{-1}$. The furnace was purged directly with air (flow rate $25 \mathrm{ml}$ $\min ^{-1}$ ) introduced through a fine glass tube inserted into the top of the oven. For experiments using humid or solvent saturated atmospheres, air was bubbled through a Dreshel bottle containing the appropriate liquid, a second empty bottle placed in-line served to prevent droplets of liquid being carried over into the oven. Sample length and temperature were stored on a microcomputer at one second intervals.

Dynamic Mechanical Analysis was performed on a TA Instruments model 983 DMA at a fixed frequency of $1 \mathrm{~Hz}$. Measurements on PET film were done using conventional sample clamps. For fibre samples, the instrument was fitted with experimental low mass dual cantilever clamps which permitted the semi-quantitative determination of the modulus and damping of unsupported fibres [10]. In order to study samples under simulated dye bath conditions a stainless steel liner was fitted inside the oven. By turning the instrument on one side and filling the liner with liquid the sample 
could be completely submerged and heated at a controlled rate without drying the samples. A heating rate of $2^{\circ} \mathrm{C} \mathrm{min}^{-1}$ was found to be satisfactory even in the presence of fluid. Stirring of the bath gave little improvement in heat transfer and was not employed due to increased noise in the instrument output.

\section{Results and Discussion}

The raw instrumental output from the TMA was analysed to determine the average sample length and amplitude of the length change over a moving window of twelve data points collected over one complete loading/unloading cycle. Typical results are shown in figure 1 for material under a dry air purge and air saturated with acetone vapour (at $22^{\circ} \mathrm{C}$ ). Softening of the samples is observed as an increase in the average length and amplitude. The apparent high temperature plateaux in the sample length curves and corresponding decrease in the amplitude curves are due to the dynamic range of the length transducer in the instrument becoming exceeded. The dynamic stress (ó) on the sample is given by the change in load $(0.1 \mathrm{~N})$ divided by the cross sectional area of the specimen. The corresponding strain (a) is similarly given by the change in length (amplitude) divided the average length. No correction is made to accommodate thinning or necking of the sample. The dynamic modulus $\left(\mathrm{E}^{*}\right)$ is obtained from ratio ó/å Unlike the more sophisticated dynamic mechanical analysis technique, the phase difference between the applied stress and corresponding strain was not monitored, thus $\mathrm{E}^{*}$ cannot be separated into storage and loss components.

Plots of dynamic moduli for cellulose acetate film run under dry (0\% RH) and humid (100\% $\mathrm{RH}$ at $20^{\circ} \mathrm{C}$ ) conditions are shown in Fig. 2. The values of $\mathrm{E}^{*}$ measured at ambient temperatures compare well with those obtained on a conventional tensile testing machine [11]. Water vapour plasticises the material - reducing its stiffness and softening temperature - acetone vapour also exerts a similar effect. These findings correlate well with anecdotal evidence from plant operatives - control of environmental conditions during processing is therefore critical to consistent production.

The effects of 2-phenoxyethanol on the damping properties of poly(ethylene terephthalate) (PET) film are shown in Fig. 3. The curves show the response of PET before treatment, during heating in 2-phenoxyethanol, re-heating in the liquid and after heating in air. During heating in 2-phenoxyethanol, very little happens until the $\mathrm{T}_{\mathrm{g}}$ of the film is reached near $100^{\circ} \mathrm{C}$ whereupon the mechanical damping (tan ä) increases dramatically as the film absorbs 2-phenoxyethanol. On reheating the swollen film, the $\mathrm{T}_{\mathrm{g}}$ of the polyester has been reduced to around $60^{\circ} \mathrm{C}$ although there is a second small maximum in tan $\ddot{a}$ at $112^{\circ} \mathrm{C}$ presumably due to regions of the polymer which have not been affected by the plasticising action of the 2-phenoxyethanol. After drying, the mechanical properties of the film are almost completely restored, although there is evidence of residual 2-phenoxyethanol causing a plasticised region (small peak in tan ä around $60^{\circ} \mathrm{C}$ ) to persist. This study helps to explain how the deep dying of PET film in this material takes place via penetration of the polymer by 2-phenoxyethanol and subsequent plasticisation of the film.

Plots of damping factor against temperature for samples of dry fibre, wet fibre alone and wet fibre treated with different levels of a model carrier (benzyl alcohol) are shown in Fig. 4. The peak in tan ä for the dry fibre corresponds to a glass transition temperature of $92^{\circ} \mathrm{C}$ which agrees closely with determinations on the same material made by DSC and TMA under comparable conditions. The $\mathrm{T}_{\mathrm{g}}$ of the wet fibre was found to be reduced to $72^{\circ} \mathrm{C}$ consistent with the findings of Hossain et $a l$. [12] who found that the diffusion coefficient of aqueous $m$-nitroaniline into acrylic fibre increased markedly at this temperature. Treatment with carrier further depresses the $\mathrm{T}_{\mathrm{g}}$ and 
broadens the damping peak characteristic of the action of a plasticiser [13]. Rinsing the fibre with hot water was found to remove the carrier from the fibre and return the $\mathrm{T}_{\mathrm{g}}$ to that of the untreated material.

The dependence of damping peak temperature on measurement frequency was studied in order to determine the apparent activation energy $\left(\ddot{\mathrm{A}} E_{\mathrm{a}}\right)$ for the glass transition process. $\ddot{\mathrm{A}} E_{\mathrm{a}}$ was found to decrease from $1000 \mathrm{~kJ} \mathrm{~mol}^{-1}$ for the dry fibre (measured around $90^{\circ} \mathrm{C}$ ) to $580 \mathrm{~kJ} \mathrm{~mol}^{-1}$ for the wet fibre $\left(\mathrm{T}_{\mathrm{g}} 70{ }^{\circ} \mathrm{C}\right)$. This parameter was found to be lower still for fibre that had been treated with carrier demonstrating the effect that plasticisation has on facilitating segmental motion. Similar effects have been reported for polyester fibres [14].

The dependence of $\mathrm{T}_{\mathrm{g}}$ (taken as the peak maximum in $\tan \delta$ at $1 \mathrm{~Hz}$ ) and concentration of dye absorbed by the fibre is shown as a function of amount of carrier added to the dye bath in Fig. 5. A maximum in the depression of $\mathrm{T}_{\mathrm{g}}$ and uptake of dye occurs around $60 \mathrm{~g} \mathrm{l}^{-1}$ of benzyl alcohol coinciding with the solubility limit of this compound in water at $80^{\circ} \mathrm{C}$. This effect has also been reported for polyester and cellulose acetate fibres $[15,16]$. Similar behaviour was found for other experimental carriers and analysis of the samples for the actual amount of carrier absorbed by the fibre shows the same relationship [17-19]. Above the saturation point of the dye bath the carrier has a greater affinity for the saturated aqueous phase than for the fibre. Consequently the degree of carrier uptake by the substrate decreases with a corresponding reduction in plasticisation and dye uptake. A plot of concentration of dye absorbed by the fibre against the reduction in $\mathrm{T}_{\mathrm{g}}$ caused by different carrier compounds is shown in Fig. 6. The solid line represents the general trend. Extrapolation of the data to the saturation point of the fibre $\left(70 \mathrm{mmol} \mathrm{kg}{ }^{-1}\right)$ suggests that a reduction in $\mathrm{T}_{\mathrm{g}}$ to around $35^{\circ} \mathrm{C}$ would be required to achieve maximum dye uptake under the chosen processing conditions.

\section{Conclusions}

Thermomechanical and Dynamic Mechanical Analysis have been shown to be suitable techniques for the determination of the softening and glass transition temperatures of fibres and films in solvents and vapours. Control of retained solvent or ambient humidity is important in the processing of cellulose acetate. The effects of 2-phenoxyethanol on poly(ethylene terephthalate) film and the influence of dye bath additives on acrylic fibres give an insight on the dyeing process. The increase in diffusional mobility brought about by a reduction in $\mathrm{T}_{\mathrm{g}}$ promotes uptake of the dye molecules into the material. For dyeing of acrylic fibres in an aqueous system, the maximum in dye uptake is found at the solubility limit of the additive in the dye bath and corresponds to the greatest reduction in $\mathrm{T}_{\mathrm{g}}$ achievable by each compound in water. These studies demonstrate that dyeing of synthetic polymers is intimately connected with the molecular mobility of the substrate.

\section{Acknowledgements}

The author would like to thank T.R. Farren (Courtaulds), D Aitken and S.M. Birkenshaw (University of Leeds) for preparing samples and supplying dye uptake data. 


\section{References}

[1] K. Meldrum and J.S. Ward, J. Soc. Dyers and Colourists, 74 (1958) 140.

[2] S. Rosenbaum, J. Appl. Polymer Sci., 9 (1965) 2071.

[3] T. Murayama and A.A. Armstrong, Jr., J. Polymer Sci. Polymer Phys. Ed., 12 (1974) 1211.

[4] A. B. Desai and G.L. Wilkes, Text. Res. J., 45 (1975) 173.

[5] W. C. Ingamells and N. Yanumet, B. Polymer J., 12 (1980) 12.

[6] M. R. Padhye and A.V. Karandikar, J. Appl. Polymer Sci., 33 (1987) 1675.

[7] J. Hermes, US Patent 4,047,889 (1977).

[8] W.C. Ingamells, R.H. Peters and S. R. Thornton, J. Appl. Polymer Sci., 17 (1973) 3733.

[9] W.C. Ingamells, J. Soc. Dyers and Colourists, 96 (1980) 466.

[10] D. Aitken, S.M. Birkenshaw, J. Catherall, R. Cox, R.E. Litchfield, D.M. Price and N.G. Todd, J. Appl. Polym. Sci.; Appl. Polym. Symp., 47 (1991) 263.

[11] T R Farren, personal communication

[12] T.M.A. Hossain, T. Iljima, Z. Morita and H. Maeda, J. Applied Polymer Sci., 13 (1969) 541.

[13] D. Aitken, S.M. Birkenshaw, J. Catherall, R. Cox and D.M. Price, J. Appl. Polym. Sci.; Appl. Polym. Symp, 47 (1991) 271.

[14] G.A. F. Roberts and R.K. Solanki, J. Soc. Dyers and Colourists, 95 (1979) 427.

[15] D. Balmforth, C.A. Bowers, J.W. Bullington, T.H. Guion and T.S. Roberts, J. Soc. Dyers \& Colourists, 82 (1966) 405.

[16] M.K. Gokhale, L. Peters and C.B. Stevens, J. Soc. Dyers and Colourists, 74 (1958) 236.

[17] D. Aitken, S.M. Burkinshaw and D.M. Price, Dyes and Pigments, 18 (1992) 23.

[18] J.P. Kim and S.M. Burkinshaw, J. Applied Polymer Sci., 49 (1993) 1647.

[19] J.P. Kim and S.M. Burkinshaw, J. Soc. Dyers and Colourists, 111 (1995) 107. 


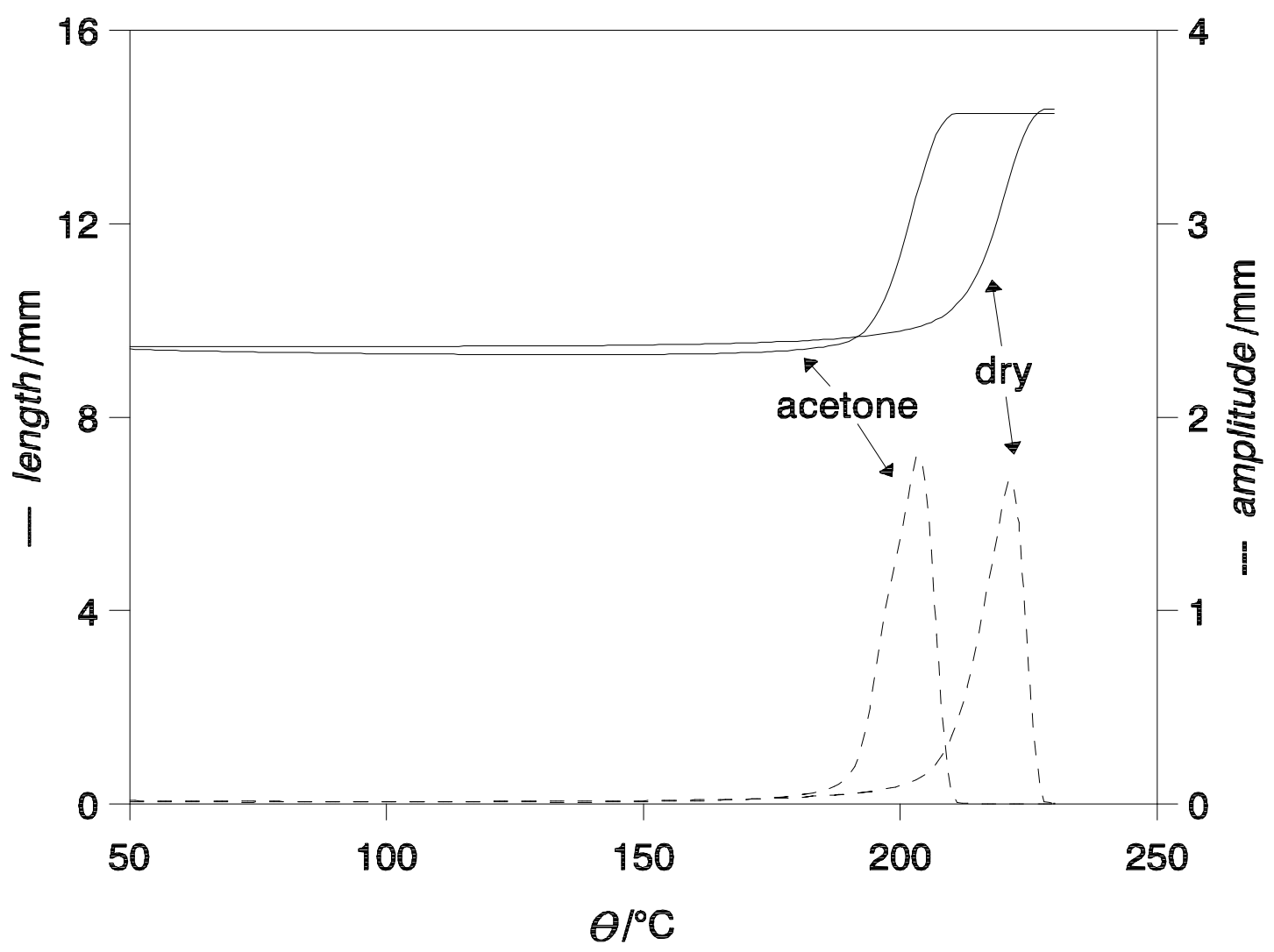

Fig.1. Length (solid line) and amplitude (dashed line) data for cellulose acetate film during dynamic loading TMA under dry and acetone saturated air purge. 


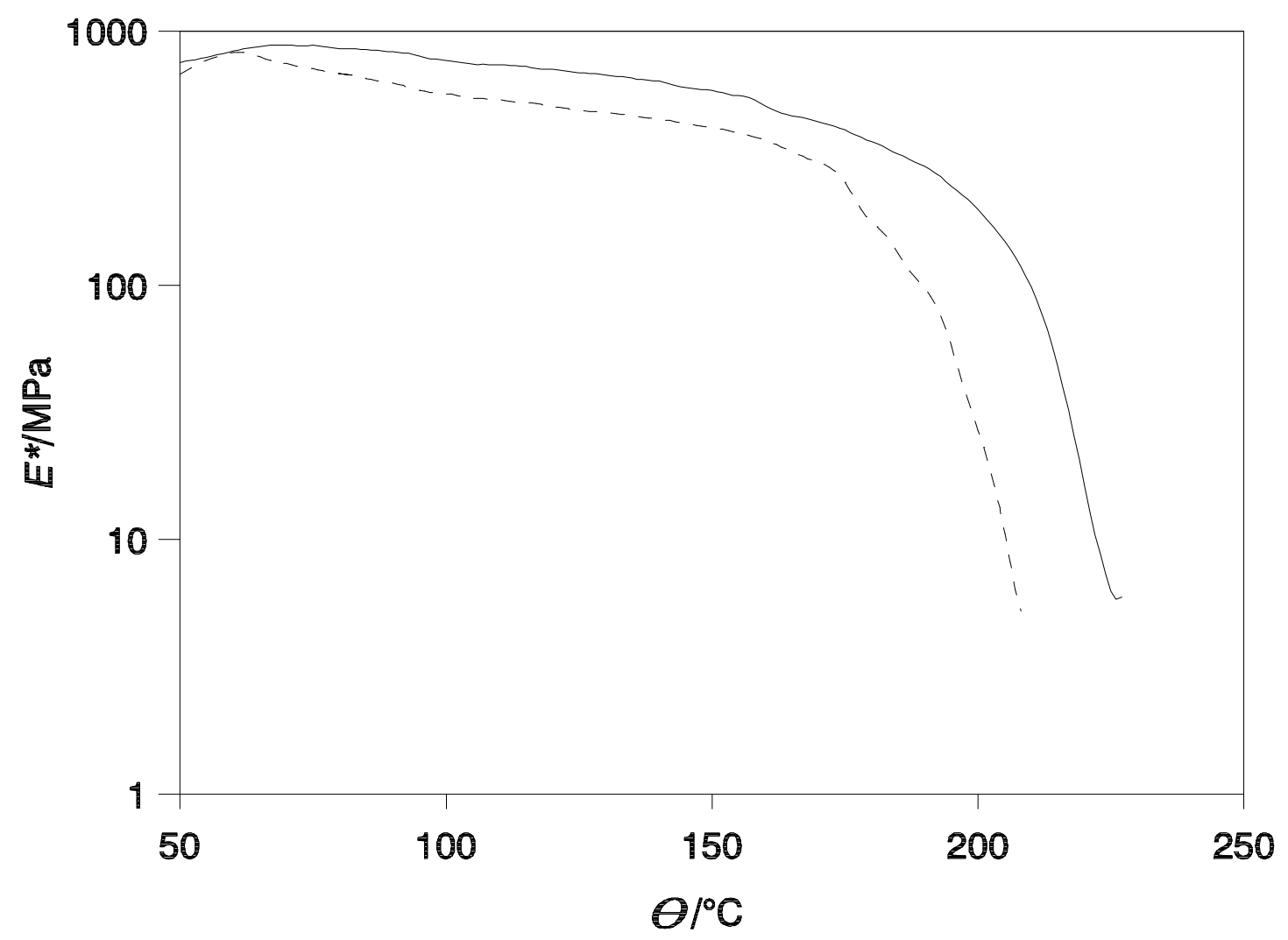

Fig. 2. Dynamic moduli of cellulose acetate films under dry (solid line) and humid (dashed line) conditions obtained from dynamic loading TMA experiments. 


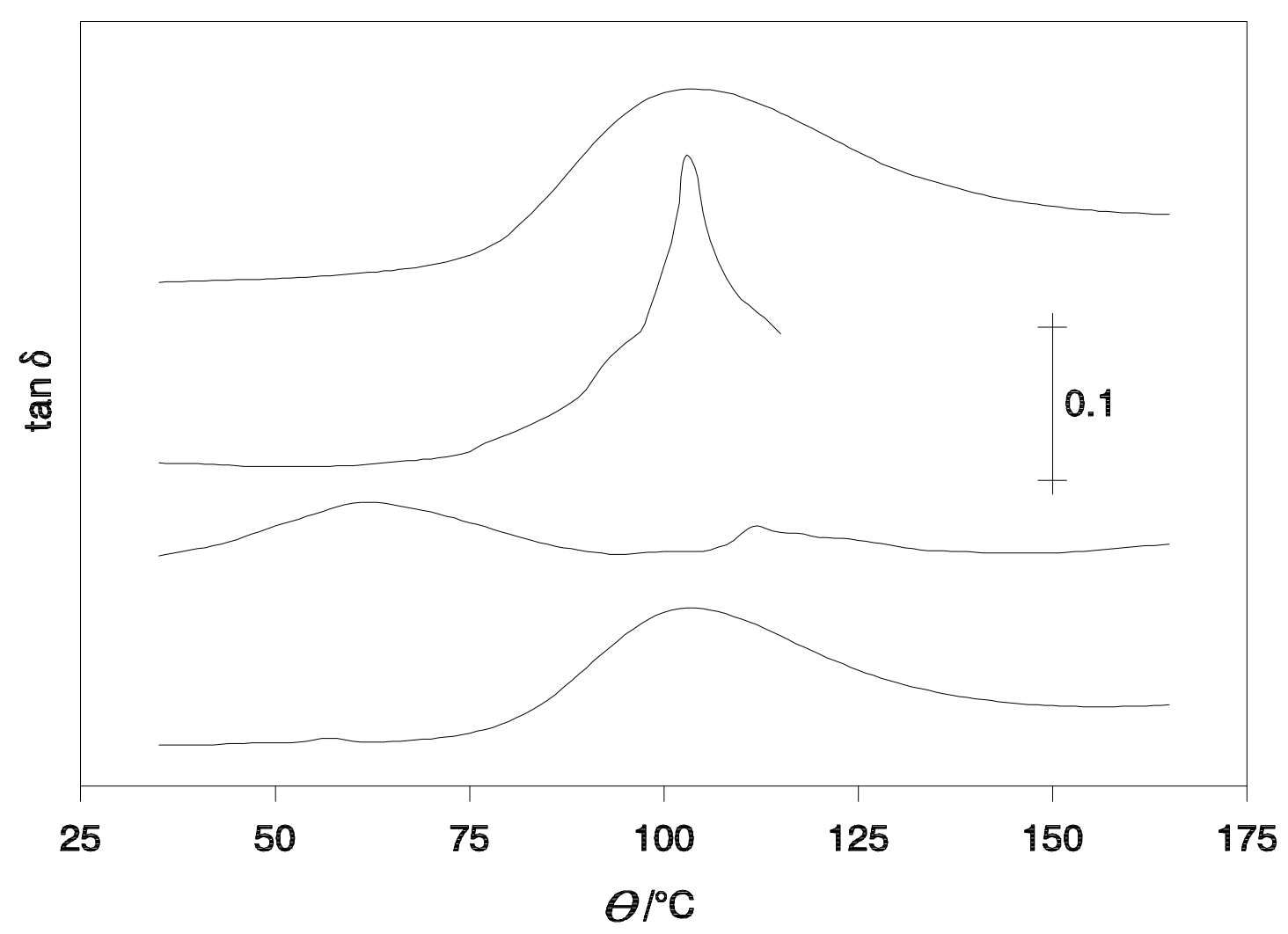

Fig. 3. Temperature dependence of the damping factor (tan ä) of PET film (top to bottom: PET before treatment, during heating in 2-phenoxyethanol, re-heating in the liquid and after heating in air). 


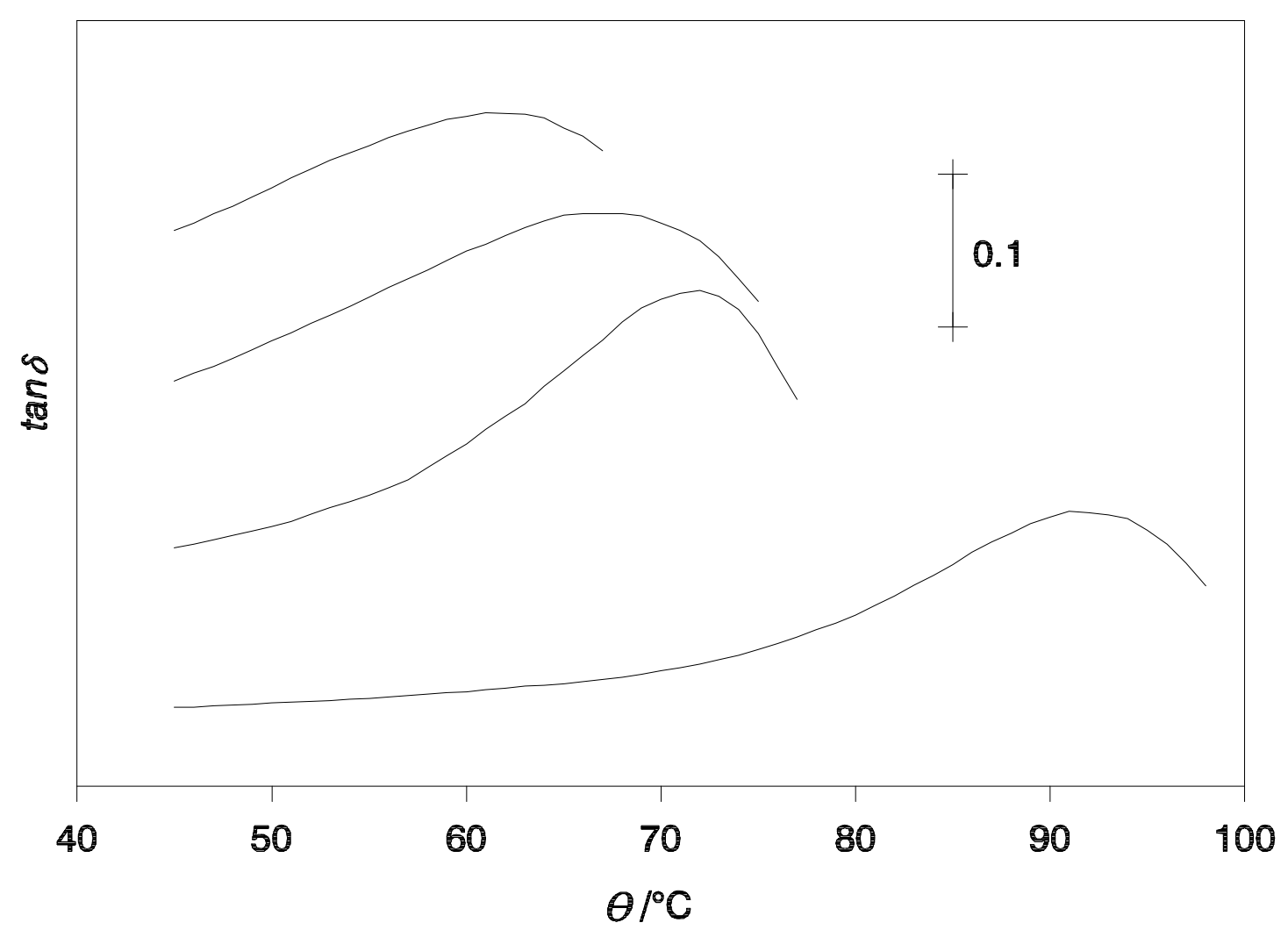

Fig.4. Temperature dependence of the damping factor (tan ä) of acrylic fibres (top to bottom: in $30 \mathrm{~g} \mathrm{l}^{-1}$ aqueous benzyl alcohol, $10 \mathrm{~g} \mathrm{l}^{-1}$ aqueous benzyl alcohol, water only and dry fibre). 


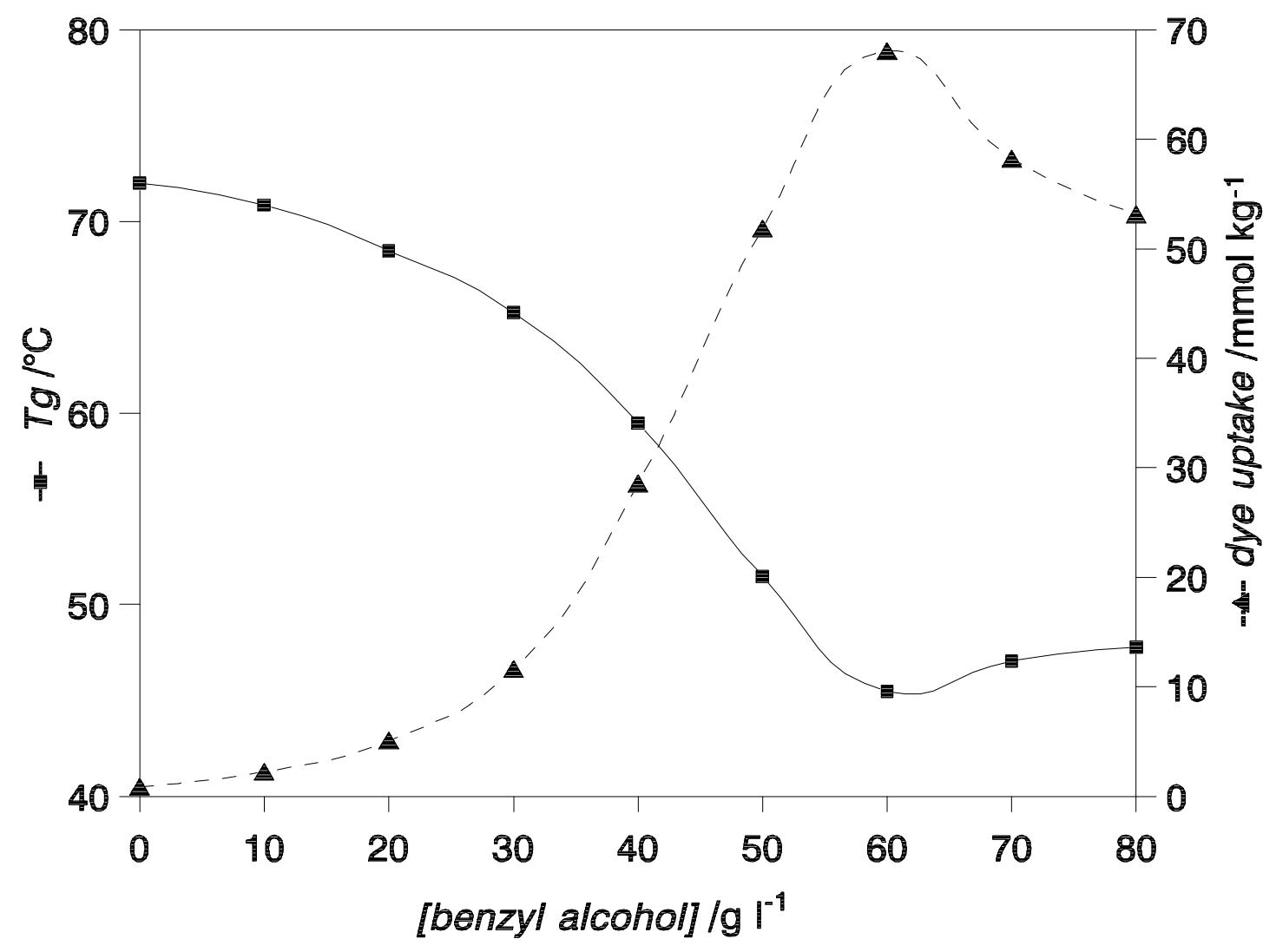

Fig. 5. Glass transition temperature (ם) and dye uptake (A) versus amount of benzyl alcohol added to the dye bath.. 


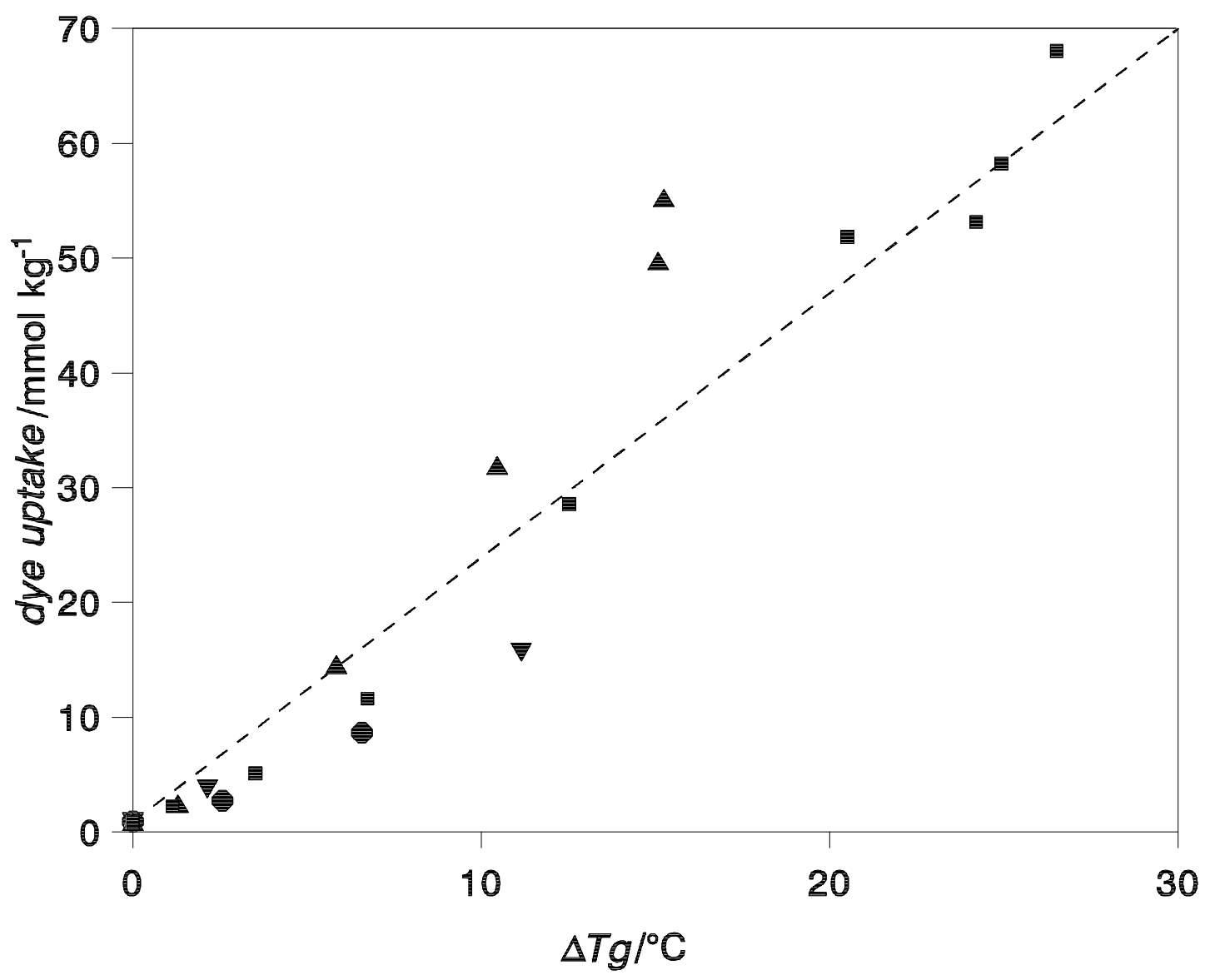

Fig. 6. Plots of dye uptake against reduction in $\mathrm{T}_{g}\left(\ddot{\mathrm{A}} \mathrm{T}_{\mathrm{g}}=\mathrm{T}_{\mathrm{g}}\right.$ [with carrier]- $\mathrm{T}_{\mathrm{g}}[$ water only]) caused by different concentrations of carriers ( $\boldsymbol{\square}$ benzyl alcohol, $\boldsymbol{\Delta}$ N-methyl formanilide, $\boldsymbol{O}$ phenethyl alcohol, $\boldsymbol{\nabla}$ 4-methyl benzyl alcohol). Dashed line indicates general trend. 